\title{
Influence of moderate maternal nutrition restriction on the fetal baboon metabolome at 0.5 and 0.9 gestation.
}

Christian Hellmuth ${ }^{\mathrm{a}}$, Olaf Uhl ${ }^{\mathrm{a}}$, Franca F. Kirchberg ${ }^{\mathrm{a}}$, Ulrike Harder ${ }^{\mathrm{a}}$, Wolfgang Peissner ${ }^{\mathrm{a}}$, Berthold Koletzko $^{\mathrm{a}}$, Peter Nathanielsz ${ }^{\mathrm{b}}$

${ }^{a}$ Ludwig-Maximilians-Universität, Division of Metabolic and Nutritional Medicine, Dr. von Hauner Children's Hospital, Universtiy of Munich Medical Center, 80337 Muenchen, Germany

${ }^{\mathrm{b}}$ Department of Obstetrics and Gynecology, Center for Pregnancy and Newborn Research, University of Texas, San Antonio, TX 78229-3900, USA

\section{Corresponding author:}

Professor Berthold Koletzko

Ludwig-Maximilians-University of Munich

Div. Metabolic and Nutritional Medicine

Dr. von Hauner Children's Hospital, University of Munich Medical Center

Lindwurmstr. 4, D-80337 Muenchen, Germany

Phone: +49 (0)89 4400 52826, Fax: +49 (0)89 440057742

Email: office.koletzko@med.lmu.de

Word count abstract: 250

Word count text: 3548

Number of figures: 3

Number of tables: 2

Number of references: 30

Keywords: Nonhuman-primate/ maternal nutrition restriction/ fetal programming/ gluconeogenesis/ metabolomics

\section{Acronyms:}

AA - amino acid; APCI - atmospheric pressure chemical ionization; BCAA - branched-chain amino acids; Carn - acylcarnitines; CTR - control group; Cys - cysteine; ESI - electrospray ionization; FA - fatty acid; G - gestation; HPLC - high-performance liquid chromatography; 
Ile - isoleucine; IUGR - intrauterine growth restriction; LC-MS - liquid chromatography coupled to MS detection; Leu - leucine; lysoPC a - lysophosphatidylcholines; Met methionine; MNR - moderate maternal nutrition restriction; MRM - multiple reaction monitoring; MS - mass spectrometer; NEFA - nonesterified fatty acids; PC aa - diacylphosphatidylcholines; PC ae - acyl-alkyl-phosphatidylcholines; PCA - principal component analysis; PEPCK - phosphoenolpyruvate carboxykinase; PGC1 $\alpha$ - peroxisome proliferatoractivated receptor gamma coactivator 1-alpha; PPARG - peroxisome proliferator activated receptor $\gamma$; QC - quality control; Ser - serine; SM - sphingomyelins; Thr - threonine; Val valine 


\section{Abstract}

\section{$\underline{\text { Background and Aims }}$}

Moderately reduced maternal nutrient availability during pregnancy has adverse effects on the fetuses' growth and metabolism during and after pregnancy. The aim of this study was to explore effects of maternal nutrition restriction (MNR) on key metabolites of the fetal energy metabolism, particularly amino acids, nonesterified fatty acids, acylcarnitines and phospholipids. These effects may reflect mechanisms relating MNR to later adverse outcomes.

\section{$\underline{\text { Methods and Results }}$}

Plasma and liver samples of fetal baboons, whose mothers were fed ad libitum (CTR) or MNR (70\% of CTR), were collected at 0.5 and 0.9 gestation ( $\mathrm{G}$ - term 184 days). Metabolites were measured with liquid chromatography coupled to mass spectrometry. In both, CTR and MNR, fetal metabolic profiles changed markedly between $0.5 \mathrm{G}$ and $0.9 \mathrm{G}$. At $0.5 \mathrm{G}$, MNR fetal plasma levels of short- and medium-chain acylcarnitines C3 ( $\mathrm{p}=0.014), \mathrm{C} 4(\mathrm{p}=0.020)$, C5 ( $\mathrm{p}=0.049), \mathrm{C} 6(\mathrm{p}=0.015), \mathrm{C} 8(\mathrm{p}=0.029)$, and $\mathrm{C} 9(\mathrm{p}=0.022)$ were elevated, but did not differ between groups at $0.9 \mathrm{G}$. Fetal liver glucose concentrations were strongly increased from $0.5 \mathrm{G}$ to $0.9 \mathrm{G}$ in $\mathrm{CTR}(\mathrm{p}=0.008)$ and $\mathrm{MNR}(\mathrm{p}=0.001)$, without differences between groups $(\mathrm{p}=0.236)$. At $0.9 \mathrm{G}$, plasma levels of methionine $(\mathrm{p}=0.015)$ and threonine $(\mathrm{p}=0.022)$, were lower in MNR group.

\section{$\underline{\text { Conclusion }}$}

Small differences in the concentrations of plasma and liver metabolites between MNR and CTR fetuses reflect good adaptation to MNR. Fetal liver metabolic profiles changed markedly between the two gestation stages, reflecting enhanced liver glucose and lipid levels with advancing gestation. Decreased concentrations of amino acids suggest an up-regulation of gluconeogenesis via this pathway in MNR. 


\section{Introduction}

Sub-optimal nutrition in pregnancy is associated with adverse metabolic offspring outcomes in later life such as reduced glucose tolerance, promotion of an atherogenic lipid profile, higher body mass index (BMI), as well as development of cardiovascular diseases [1]. These outcomes are generally attributed to developmental programming which can be defined as the response to a specific challenge to the mammalian organism during a critical developmental time window [2]. This may alter the trajectory of development, modifying the organism's phenotype with effects on health that can persist throughout the life-course [3]. Several different animal models have been used to examine the effect of reduced maternal nutrition on metabolic disorders. Most studies have been conducted in rodents $[4,5]$ which cannot simply be extrapolated to humans that have a very different developmental profile from monotocous, precocial species [6]. Thus, studies with nonhuman primates are required in order to understand early development of metabolic diseases in humans. In baboons, moderate maternal nutrition restriction (MNR) has only minor effects on overall fetal growth at 0.5 gestation ( $\mathrm{G}$ - term 184 days) [7,8], but at $0.9 \mathrm{G}$ fetuses show intrauterine growth restriction

[9]. Decreased maternal nutrition in pregnant baboons was shown to alter the placental nutrient transfer [10], fetal metabolism [7] and was associated with an increased risk for postnatal adverse outcomes such as insulin resistance [11]. Metabolic adaptations during development in response to decreased fetal nutrition may persist after birth, even when nutrition has returned to the normal range [12]. For instance, fetuses experiencing MNR have increased phosphoenolpyruvate carboxykinase (PEPCK) activity in the fetal liver, indicating elevated gluconeogenesis [13] and they also have higher fasting glucose levels 3.5 years after birth [11]. The aim of the present study was to explore the effects of MNR in pregnancy on the metabolic signature of fetal baboons. Amino acids (AA) are precursors for endogenous gluconeogenesis. Most of the AA are actively transported across the placenta to enter the fetal 
circulation to support fetal growth [14]. Lipids or fatty acids (FA) are released from placenta into the fetal circulation in the form of nonesterified fatty acids (NEFA) and are esterified in phospholipids by the fetal liver [15]. For this study, we used a subset of samples from a previously published fetal baboon study and analyzed AA, NEFA, and polar lipids (acylcarnitines and phospholipids). 


\section{Material and methods}

\section{Ethics statement}

All procedures performed in studies involving animals were in accordance with the ethical standards of the University of Texas Health Science Center and Southwest National Primate Research Center Institutional Animal Care and Use Committees. All applicable international, national, and/or institutional guidelines for the care and use of animals were followed.

\section{Animal care}

Twenty-seven baboons (Papio hamadryas anubis) from the Southwest National Primate Research Center, San Antonio Texas were studied (Table 1). Animals were housed in outdoor gang cages providing full social and physical activity [16]. Animals were fed between 7am and $9 \mathrm{am}$ or $11 \mathrm{am}$ and $1 \mathrm{pm}$ as described [16]. Water was continuously available in the feeding cages via individual waterers (Lixit, Napa, CA). Animals received Monkey Diet 15\% 5LE0 ( $\geq 15 \%$ crude protein, $\geq 4 \%$ crude fat, $\geq 10 \%$ crude fiber; Purina, St. Louis, MO). At the start of the feeding period, ad libitum-fed baboons were given 60 biscuits in their individual cage. Biscuits remaining were counted after baboons returned to their group cage.

\section{Study}

Healthy non-pregnant female baboons of similar body weights $(10-15 \mathrm{~kg})$ were randomly assigned to one of two group cages in social groups of 10-16 animals, and a vasectomized male. After acclimation to the feeding cages ( $30 \mathrm{~d})$, a fertile male was substituted. Pregnancy was dated initially by timing of ovulation and changes in sex skin color, and confirmed by 
ultrasound at $30 \mathrm{~d}$ of gestation. From this day on, 12 of the 27 pregnant baboons were fed $70 \%$ of food eaten by contemporaneous controls on a per-kilogram basis (maternal nutrition restriction, MNR), while the remaining 15 pregnant baboons were still ad libitum-fed (control, CTR). Before pregnancy, the two groups did not differ in morphometric characteristics [17] (Table 1).

Standard cesarean sections were performed at $0.5 \mathrm{G}$ and $0.9 \mathrm{G}$ (term 184 days) after an overnight fast. The umbilical cord was identified and elevated to the incision to enable fetal exsanguination, as approved by the American Veterinary Medical Association. The fetus was removed from the uterus and immediately submitted for morphometric measurements, complete pathologic evaluation, and tissue sampling. The complete fetal liver was removed and half of the sample was taken for this study and immediately flash frozen in liquid nitrogen [18].

\section{Preparation of liver tissue}

Briefly, an aliquot of about $50 \mathrm{mg}$ of the liver tissue was diluted with $600 \mu 1$ methanol and bead-based homogenized in cryo-vials with a MINILYS homogenizer (Precellys, Bertin Technologies, Montigny-le-Bretonneux, France). Subsequently, samples were centrifuged for $10 \mathrm{~min}$ at $23306 \mathrm{~g}$ (room temperature). $20 \mu \mathrm{l}$ of the supernatant were transferred and processed as described for plasma and standards.

\section{Determination of metabolites}

For analysis of polar lipids, serum, liver extract, and standard solutions were diluted with methanol. After centrifugation, supernatants were injected into a liquid chromatographic system (Agilent 1200 SL series, Waldbronn, Germany) coupled to a triple quadrupole mass 
spectrometer (4000 QTRAP, AB Sciex, Darmstadt, Germany) with an electrospray ionization (ESI) source. The system was used as flow-injection analysis and the mass spectrometer (MS) was run in Multiple Reaction Monitoring (MRM) mode in positive and negative ionization mode. The analysis was performed for acylcarnitines (Carn), diacyl-phosphatidylcholines (PCaa), acyl-alkyl-phosphatidylcholines $\quad$ (PCae), sphingomyelines $\quad(\mathrm{SM})$, lysophosphatidylcholines (lysoPCa) and sum of hexoses, hereafter referred to as glucose which accounts for more than $80 \%$ of this value.

AA analysis was performed as reported previously [19]. Serum, liver extract, and standard solutions were prepared by derivatisation to amino acid butylester, and determined by LCMS/MS with MRM mode and an atmospheric pressure chemical ionization source operating in positive ionization mode.

Analysis of NEFA was performed as previously reported [20]. Briefly, serum, liver extract, and standard solutions were mixed with isopropanol. After centrifugation the supernatant was used for LC-MS/MS analysis operating with MRM in negative ESI mode.

The entire analytical process was post-processed by the Analyst 1.5.1 and R software. The polar lipids and NEFA are mentioned as $\mathrm{CX}$ :Y. In this nomenclature, $\mathrm{X}$ is the length of the carbon chain, $\mathrm{Y}$ is the number of double bonds, $\mathrm{OH}$ in the formula indicates that the molecule contains a hydroxyl-group. "a" indicates that the acyl chain is bound via an ester bond to the backbone, while "e" indicates binding by an ether bound. We report all metabolite concentrations in $\mu \mathrm{mol} / \mathrm{L}$ serum and $\mu \mathrm{mol} / \mathrm{kg}$ liver. Sums of AA, Carn, NEFA, lysoPCa, PCaa, PCae, and SM were calculated by adding up all analytes of the groups, respectively. Sum of all phosphatidylcholines (PC) is the sum of all PCaa and PCae. The sum of branchedchain AA (BCAA) is the sum of Leucine (Leu), Isoleucine (Ile), and Valine (Val). The sum of the short chain acylcarnitines was calculated by adding Carn C3, C4, and C5. Furthermore, 12 
different ratios were calculated by using the absolute concentrations of the selected metabolites.

\section{Data analysis}

Statistical data analyses were performed using R (programming language, version 3.0.1).

To quantify the measurement accuracy, six plasma quality control (QC) samples were consistently measured along with the samples. Analytes with a coefficient of variation higher than $15 \%$ were excluded from the statistical analyses. Furthermore, analytes with more than $20 \%$ missing values were excluded. Outlier analysis was performed for each compartment (liver, plasma) and each gestational stage $(0.5 \mathrm{G}, 0.9 \mathrm{G})$ separately. For each analyte, we determined the interval between the two highest values and in case this interval was higher than one standard deviation of the metabolite's concentration, the higher sample was defined as outlier. If a sample was defined as outlier for more than $25 \%$ of the analytes of one of the three applications (AA, NEFA, polar lipids), the sample was excluded.

Principal component analysis (PCA) was performed to identify possible grouping of samples according the treatment, collection time point, or compartment. For group and time point comparisons, we calculated exact non-parametric permutations tests which are well suited to small sample sizes. The significance level was set at 0.05 . Bonferroni-corrected p-values can be obtained by multiplying the reported p-values with the factor 206 (number of analytes determined). Results are visualized using Manhattan plots, where the $\log _{10}$ transformed pvalues (y-axis) are plotted against the analytes (x-axis). If the median was higher in the MNR group or at $0.9 \mathrm{G}$, the positive value of the log transformed p-value was plotted. Otherwise, the negative value was plotted. 


\section{Results}

After detection of outliers as described above, two fetal liver samples of different groups were excluded from all statistical analyses (Table 1). We assume an error during the preparation of the liver samples. Two hundred-six different metabolites were determined in the fetal plasma samples (Supplemental Table 1). Out of these, 195 were determined in fetal liver (Supplemental Table 2).

\subsection{G vs. 0.9G}

The PCA illustrates a strong grouping by the nature of the sample, either fetal plasma or fetal liver (Supplemental Fig.1), and stage of gestation, but there was no grouping according to the different diets. The results of the PCA were confirmed by permutation tests (Table 2, Supplemental Tables $1 \& 2$ ). In both feeding groups, fetal liver levels of polyunsaturated NEFA, histidine, polyunsaturated PCaa and polyunsaturated PCae were significantly higher at 0.9G, while NEFA 26:1 and 26:2, aspartic acid, glutamic acid and glycine, saturated PCaa and saturated PCae were significantly lower at $0.9 \mathrm{G}$ compared to 0.5G. Comparing glucose levels in fetal liver between $0.5 \mathrm{G}$ und $0.9 \mathrm{G}$, a strong increase of glucose both in the CTR group (from 60 to $2013 \mathrm{mmol} / \mathrm{kg}$ ) and in MNR fetuses (from 51 to $2515 \mathrm{mmol} / \mathrm{kg}$ ) with age was observed.

In fetal plasma, polyunsaturated NEFA were lower, while polyunsaturated PCae were higher at $0.9 \mathrm{G}$ than at $0.5 \mathrm{G}$ in both feeding groups.

\section{MNR vs CTR}


At $0.5 \mathrm{G}$, MNR fetal plasma levels of the short-chain acylcarnitines Carn C3, Carn C4, Carn C5, Carn C6, Carn C8, and Carn C9 were higher compared to those of the CTR group (Fig. 2, Table 2). Furthermore, PCaa C36.2, PCae C36, PCae C36.2, PCae C38:2, SM C34:2, SM C36:2, SM C42:3, $\mathrm{SM}(\mathrm{OH}) \mathrm{C} 34: 2$, and $\mathrm{SM}(\mathrm{OH}) \mathrm{C} 38: 2$ were significantly higher at $0.5 \mathrm{G}$ in the MNR group compared to the CTR group. The sum of short chain Carn, the ratio of Carn C4/Val, the ratio of Carn C3/Ile, and the ratio of the sum of short chain Carn to BCAA were significantly higher in the MNR group compared to CTR in fetal plasma (Table 2). Most interestingly, no metabolite was lower in the MNR group at $0.5 \mathrm{G}$, neither in fetal liver nor in fetal plasma (Table 2).

$0.9 G$

At $0.9 \mathrm{G}$, the differences of short-chain acylcarnitines between groups were no longer present in fetal plasma (Fig. 2). The short-chain acylcarnitines C2, C3, C4, C6, and C9 decreased significantly from $0.5 \mathrm{G}$ to $0.9 \mathrm{G}$ in fetal plasma of the MNR group (Table 2), but not in the control group. Additionally, we found lower fetal plasma levels of methionine (Met) and threonine (Thr) as well as lysoPCa C20:4, and NEFA 26:5 in the MNR group compared to CTR group at 0.9G (Fig.2, Table 2). In the fetal liver samples, concentrations of NEFA 26:1 and Thr were lower in the MNR group at 0.9G while Carn C12:1, PCaa C34:1, PCaa C38:3, PCae C34:1, PCae C44:4, and $\mathrm{SM}(\mathrm{OH}) \mathrm{C} 42: 4$ were higher compared to the CTR group (Table 2). The ratios of Carn C3 to Thr, to Val, and to Met as well as Cysteine (Cys) to Met were significantly higher in liver of the MNR group at 0.9G (Table 2). Furthermore, the ratios of Carn C3 to Met, Thr, Serine (Ser), Val, and Ile only increased significantly in the MNR group (Fig.3). The total amounts of fetal plasma lysoPCa, PCaa, PCae, and SM increased 
significantly from $0.5 \mathrm{G}$ to $0.9 \mathrm{G}$ in fetal plasma of the MNR group, but not in the CTR group (Table 2). 


\section{Discussion}

\subsection{G vs. 0.9G}

The differences between the metabolic profiles at $0.5 \mathrm{G}$ and $0.9 \mathrm{G}$ detected in both MNR and CTR reflect the development of fetal metabolism. The marked increase of fetal liver glucose between $0.5 \mathrm{G}$ and $0.9 \mathrm{G}$ indicates an increased gluconeogenesis in both feeding groups. This finding might explain the results by Li et al. (2009) who found that liver glycogen was higher in the MNR than CTR fetuses [21]. This increase was shown to be due to an enhanced activity of PEPCK which results from decreased methylation of the gene's promoter [13]. Formerly, it was assumed that substantial gluconeogenesis only starts to occur` at birth [22], but more recent publications, using mammalian animal models of intrauterine growth restriction and inadequate maternal nutrition, point towards intra-uterine initiation of gluconeogenesis [13, 23, 24]. In fetal baboons it was shown that the PEPCK protein, a key enzyme in gluconeogenesis, was upregulated in MNR fetuses [13]. Probably, the elevated gluconeogenesis occurs just in MNR, but this shows that the involved enzymes are sensitive to environmental changes, and thus the mechanisms should be available also in the CTR group. However, since fetal gluconeogenesis is particularly shown in MNR and IUGR animal models (see below), elevation of glucose in CTR may also occur by increased placental transport and enrichment in the fetal liver. The unchanged plasma glucose levels between $0.5 \mathrm{G}$ and $0.9 \mathrm{G}$ may be explained by a balance of glucose supply by the placenta and demand by liver and muscle. Further studies in fetal non-human primates should therefore include analysis of hormones, gene expression, or activity of enzymes involved in gluconeogenesis. We also found indications for an elevated hepatic lipid uptake. Higher levels of fetal hepatic polyunsaturated NEFA at $0.9 \mathrm{G}$ compared to $0.5 \mathrm{G}$ and lower levels of plasma polyunsaturated 
NEFA indicate a stronger absorption by the liver for increased lipid synthesis or energy demand [25].

\section{MNR vs CTR}

Although the MNR mothers received fewer amounts of all macronutrients, including lipids in their diet, the hepatic NEFA levels were not different between MNR and CTR fetuses. The changes over time were similar in both treatment groups, indicating that both MNR mothers and fetuses are able to adapt to the lower nutritional plane. The strength of adaptation of the maternal-fetal system to the moderate nutrition restriction during pregnancy is reflected by the lack of decreased metabolites in the MNR group at $0.5 \mathrm{G}$. This points towards a metabolic adaption already in the first half of pregnancy, most likely reflecting compensatory adaption of maternal metabolism, placental nutrient transfer, and/or fetal metabolism. This is in line with the results published by McDonald et al. who reported no difference in maternal and only small differences of fetal amino acids at $0.5 \mathrm{G}$ in nutrient restricted baboons [8].

Elevated short- and medium-chain Carn levels in fetal plasma of MNR fetuses at 0.5G may reflect increased oxidation of AA [26] or FA in peroxisomes [27]. The sum of short-chain Carn and several ratios quantifying BCAA degradation were significantly higher in the MNR group compared to CTR, which supports the hypothesis of an enhanced BCAA degradation. Another explanation would be increased placental transfer of ketone-bodies which are subsequently degraded to short-chain FA intermediates for energy provision [25]. At 0.9G, the differences of short-chain Carn between the groups were no longer present in fetal plasma. Since both groups showed a similar increase in liver glucose, it is clear that MNR fetuses had up-regulated adaptive mechanisms between 0.5 and 0.9G. Previously, it has been demonstrated that cortisol and PEPCK are increased in fetal liver of MNR fetuses [13] pointing towards a higher gluconeogenesis rate of MNR fetuses at $0.9 \mathrm{G}$ compared to controls. 
Plasma cortisol, a key regulator of PEPCK, is also increased in late-gestation in female offspring born to nutrient-restricted ewes [28]. In vitro effects of glucocorticoids and PEPCK in isolated fetal baboon hepatocytes have been shown by Li et al. [29]. Thus, many facts speak in favor of an elevated gluconeogenesis rate in MNR fetuses and we assume a utilization of AA for this elevated gluconeogenesis rate. At $0.9 \mathrm{G}$, we found lower fetal plasma levels of Met and Thr as well as lower fetal liver levels of Thr. Met and Thr are transformed via propionyl-CoA and succinyl-CoA pathway to glucose. The ratios of Carn $\mathrm{C} 3$ to $\mathrm{Thr}$, to Val, and to Met were higher in the liver of the MNR group at $0.9 \mathrm{G}$ and increased only in the MNR group from to $0.5 \mathrm{G}$ to $0.9 \mathrm{G}$. The enhanced gluconeogenesis in MNR fetuses may be explained by an up-regulated degradation of AA to propionyl-CoA (Carn C3) which is transformed to succinyl-CoA, phosphoenol pyruvate, and finally glucose. The programming effect of this enhanced rate of gluconeogenesis provides potential benefits to the fetus during periods of under-nutrition during pregnancy. Elevated gluconeogenesis may explain the emergence of insulin resistance in infant baboons exposed to this degree of under-nutrition as fetuses $[11,24]$. Other explanations for the behavior of these amino acids may be adaptions in placental transport or utilization for elevated ketogenesis in MNR mothers, although other AA should also be affected by alterations in ketone body production. Since fetuses probably have higher energy demand in the second half of gestation, we assumed more difference in the metabolic profiles at $0.9 \mathrm{G}$. However, with our metabolomics approach we detected only small difference between MNR and CTR in late gestation. A possible explanation might be the small sample number. For ethical reasons, we chose an existing fetal baboon study with the aim to raise hypotheses. The small sample number reduces the power and herewith the chance of detecting a true effect and to use more sophisticated statistical methods, like cluster analysis. Despite the novel findings of the presented study, there are some limitations. The biggest limitation is the unequal distribution of confounding factors. Particularly, fetal sex 
differences cannot be adjusted for in this study. Cox et al. reported that adaptions in the placenta were only found in female placentas and not in male placentas or without accounting for fetal sex [30].

Summarized, we found only small differences in the plasma and liver metabolites pool between MNR and CTR fetuses, indicating a good adaptation of the mother and/or the fetus to the moderate maternal nutrition restriction. We were also able to show that for different stages of gestation, different metabolic mechanisms were altered in order to adapt to the decreased maternal nutrient supply. These results indicate possible mechanisms by which fetuses can adapt to moderate maternal nutrition restriction. Furthermore, we showed strongly altered fetal metabolism at $0.9 \mathrm{G}$ compared to $0.5 \mathrm{G}$, in particular increased liver glucose and lipogenesis. The compensatory mechanism in MNR fetuses, maintaining the same lipo- and gluco-homeostasis compared to CTR, may represent the programming mechanisms leading to a higher glucose intolerance in MNR infants in later life.

\section{Acknowledgments}

This work was financially supported by the Commission of the European Communities, the 7th Framework Programme, contract FP7-289346-EARLY NUTRITION, and the European Research Council Advanced Grant ERC-2012-AdG - no.322605 META-GROWTH. This manuscript does not necessarily reflect the views of the Commission and in no way anticipates the future policy in this area. The funders had no role in study design, data collection and analysis, decision to publish, or preparation of the manuscript. The authors declare to have no conflict of interest. 


\section{References}

[1] Roseboom TJ, van der Meulen JH, Ravelli AC, Osmond C, Barker DJ, Bleker OP. Effects of prenatal exposure to the Dutch famine on adult disease in later life: an overview. Mol Cell Endocrinol. 2001;185:93-8.

[2] Koletzko B, Brands B, Chourdakis M, Cramer S, Grote V, Hellmuth C, et al. The Power of Programming and The Early Nutrition Project: opportunities for health promotion by nutrition during the first thousand days of life and beyond. Ann Nutr Metab 2014;64:141-50.

[3] Nathanielsz PW, Ford SP, Long NM, Vega CC, Reyes-Castro LA, Zambrano E. Interventions to prevent adverse fetal programming due to maternal obesity during pregnancy. Nutrition reviews. 2013;71 Suppl 1:S78-87.

[4] Ozanne SE, Hales CN. The long-term consequences of intra-uterine protein malnutrition for glucose metabolism. The Proceedings of the Nutrition Society. 1999;58:615-9.

[5] Remacle C, Bieswal F, Bol V, Reusens B. Developmental programming of adult obesity and cardiovascular disease in rodents by maternal nutrition imbalance. Am $\mathrm{J}$ Clin Nutr. 2011;94:1846S-52S.

[6] Rabadan-Diehl C, Nathanielsz P. From Mice to Men: research models of developmental programming. Journal of developmental origins of health and disease. 2013;4:3-9.

[7] Li C, Schlabritz-Loutsevitch NE, Hubbard GB, Han V, Nygard K, Cox LA, et al. Effects of maternal global nutrient restriction on fetal baboon hepatic insulin-like growth factor system genes and gene products. Endocrinology. 2009;150:4634-42.

[8] McDonald TJ, Wu G, Nijland MJ, Jenkins SL, Nathanielsz PW, Jansson T. Effect of 30\% nutrient restriction in the first half of gestation on maternal and fetal baboon serum amino acid concentrations. Br J Nutr. 2013;109:1382-8.

[9] Li C, McDonald TJ, Wu G, Nijland MJ, Nathanielsz PW. Intrauterine growth restriction alters term fetal baboon hypothalamic appetitive peptide balance. J Endocrinol. 2013;217:275-82.

[10] Kavitha JV, Rosario FJ, Nijland MJ, McDonald TJ, Wu G, Kanai Y, et al. Downregulation of placental mTOR, insulin/IGF-I signaling, and nutrient transporters in response to maternal nutrient restriction in the baboon. FASEB J. 2014;28:1294-305.

[11] Choi J, Li C, McDonald TJ, Comuzzie A, Mattern V, Nathanielsz PW. Emergence of insulin resistance in juvenile baboon offspring of mothers exposed to moderate maternal nutrient reduction. Am J Physiol Regul Integr Comp Physiol. 2011;301:R757-62.

[12] Symonds ME, Sebert SP, Hyatt MA, Budge H. Nutritional programming of the metabolic syndrome. Nat Rev Endocrinol. 2009;5:604-10.

[13] Nijland MJ, Mitsuya K, Li C, Ford S, McDonald TJ, Nathanielsz PW, et al. Epigenetic modification of fetal baboon hepatic phosphoenolpyruvate carboxykinase following exposure to moderately reduced nutrient availability. J Physiol. 2010;588:1349-59.

[14] Battaglia FC, Regnault TR. Placental transport and metabolism of amino acids. Placenta. 2001;22:145-61.

[15] Haggarty P. Fatty acid supply to the human fetus. Annu Rev Nutr. 2010;30:237-55.

[16] Schlabritz-Loutsevitch NE, Howell K, Rice K, Glover EJ, Nevill CH, Jenkins SL, et al. Development of a system for individual feeding of baboons maintained in an outdoor group social environment. J Med Primatol. 2004;33:117-26.

[17] Nijland MJ, Schlabritz-Loutsevitch NE, Hubbard GB, Nathanielsz PW, Cox LA. Nonhuman primate fetal kidney transcriptome analysis indicates mammalian target of rapamycin (mTOR) is a central nutrient-responsive pathway. J Physiol. 2007;579:643-56. 
[18] Schlabritz-Loutsevitch NE, Hubbard GB, Dammann MJ, Jenkins SL, Frost PA, McDonald TJ, et al. Normal concentrations of essential and toxic elements in pregnant baboons and fetuses (Papio species). J Med Primatol. 2004;33:152-62.

[19] Harder U, Koletzko B, Peissner W. Quantification of 22 plasma amino acids combining derivatization and ion-pair LC-MS/MS. J Chromatogr B Analyt Technol Biomed Life Sci. 2011;879:495-504.

[20] Hellmuth C, Weber M, Koletzko B, Peissner W. Nonesterified Fatty Acid Determination for Functional Lipidomics: Comprehensive Ultrahigh Performance Liquid ChromatographyTandem Mass Spectrometry Quantitation, Qualification, and Parameter Prediction. Analytical Chemistry. 2012.

[21] Li C, Levitz M, Hubbard GB, Jenkins SL, Han V, Ferry RJ, Jr., et al. The IGF axis in baboon pregnancy: placental and systemic responses to feeding $70 \%$ global ad libitum diet. Placenta. 2007;28:1200-10.

[22] Kalhan S, Parimi P. Gluconeogenesis in the fetus and neonate. Semin Perinatol. 2000;24:94-106.

[23] Lie S, Morrison JL, Williams-Wyss O, Suter CM, Humphreys DT, Ozanne SE, et al. Impact of embryo number and maternal undernutrition around the time of conception on insulin signaling and gluconeogenic factors and microRNAs in the liver of fetal sheep. Am $\mathbf{J}$ Physiol Endocrinol Metab. 2014;306:E1013-24.

[24] Thorn SR, Brown LD, Rozance PJ, Hay WW, Jr., Friedman JE. Increased hepatic glucose production in fetal sheep with intrauterine growth restriction is not suppressed by insulin. Diabetes. 2013;62:65-73.

[25] Herrera E, Amusquivar E. Lipid metabolism in the fetus and the newborn. Diabetes Metab Res Rev. 2000;16:202-10.

[26] Roe DS, Roe CR, Brivet M, Sweetman L. Evidence for a short-chain carnitineacylcarnitine translocase in mitochondria specifically related to the metabolism of branchedchain amino acids. Mol Genet Metab. 2000;69:69-75.

[27] Schooneman MG, Vaz FM, Houten SM, Soeters MR. Acylcarnitines: reflecting or inflicting insulin resistance? Diabetes. 2013;62:1-8.

[28] George LA, Zhang L, Tuersunjiang N, Ma Y, Long NM, Uthlaut AB, et al. Early maternal undernutrition programs increased feed intake, altered glucose metabolism and insulin secretion, and liver function in aged female offspring. Am J Physiol Regul Integr Comp Physiol. 2012;302:R795-804.

[29] Li C, Shu ZJ, Lee S, Gupta MB, Jansson T, Nathanielsz PW, et al. Effects of maternal nutrient restriction, intrauterine growth restriction, and glucocorticoid exposure on phosphoenolpyruvate carboxykinase-1 expression in fetal baboon hepatocytes in vitro. $\mathrm{J}$ Med Primatol. 2013;42:211-9.

[30] Cox LA, Li C, Glenn JP, Lange K, Spradling KD, Nathanielsz PW, et al. Expression of the placental transcriptome in maternal nutrient reduction in baboons is dependent on fetal sex. J Nutr. 2013;143:1698-708. 


\section{Figure legends}

Fig.1 Score plot of the first two Principle Components (PC) of the Principal Component Analysis (PCA) of fetal liver (a) and fetal plasma (b). Each point represents one sample and is labeled according to sample type and gestational age. Circles represent samples collected from fetuses of control group (CTR) and triangles fetuses of maternal nutrient restricted group (MNR). During the laboratory process, sample number of liver samples was reduced.

Fig.2 Manhattan plot for permutation tests at 0.5 gestation (G) (a) and 0.9G (b) fetal plasma between maternal nutrition restriction (MNR) and control group (CTR). AA - amino acid; BCAA - branched-chain amino acids; Carn - acylcarnitines; CTR - control group; G gestation; lysoPC a - lysophosphatidylcholines; MNR - moderate maternal nutrition restriction; NEFA - nonesterified fatty acids; PC aa - diacyl-phosphatidylcholines; PC ae acyl-alkyl-phosphatidylcholines; SM - sphingomyelins

Fig.3 Ratios of acylcarnitine (Carn) C3 to methionine (Met), threonine (Thr), serine (Ser), valine (Val), and isoleucine (Ile) at 0.5 and 0.9 gestation $(\mathrm{G})$ in control group (CTR, rhomb) and maternal nutrition restriction group (MNR, circle) in fetal liver. The crosses indicate the position of the respective medians. An asterisk over the dashed line stands for a significant difference between the stages of gestation. An asterisk beside a vertical line connecting the medians of the same stage of gestation stands for a significant difference between the groups at this time point. Significance was determined using permutation tests. $* \mathrm{p}<0.05$, $* * \mathrm{p}<0.01$, $* * * \mathrm{p}<0.001$ 


\section{Tables}

Table 1 Characteristics of the studied baboon population for the maternal nutrient restricted group (MNR) and control group (CTR) at 0.5 and 0.9 gestation (G).

\begin{tabular}{|l|c|c|c|c|}
\hline \multirow{2}{*}{} & \multicolumn{2}{|c|}{ CTR } & \multicolumn{2}{|c|}{ MNR } \\
\cline { 2 - 5 } & $\mathbf{0 . 5 G}$ & $\mathbf{0 . 9 G}$ & $\mathbf{0 . 5 G}$ & $\mathbf{0 . 9 G}$ \\
\hline Number (used for complete analysis) & $6(5)$ & 9 & 8 & $4(3)$ \\
Fetal Sex [male] (used for complete analysis) & $4(3)$ & 3 & 3 & $2(1)$ \\
Maternal age at CS [years] & $11.25[4.96]$ & $8.25[1.5]$ & $8.46[1.38]$ & $12.67[1]^{*}$ \\
Maternal weight [g] & $14.22[2.5]$ & $16.97[2.57]$ & $14.49[2.88]$ & $15.39[1.13]$ \\
Maternal weight at CS [g] & $13.89[2.39]$ & $17.75[3.12]$ & $14.21[3.16]$ & $15.6[1.26]^{*}$ \\
Fetal weight [g] & $101.1[7.58]$ & $737.7[182.5]$ & $104.65[14.58]$ & $693[71.75]$ \\
Total storage time [years] & $5.8[5.38]$ & $2.42[4.25]$ & $3.5[5.08]$ & $6.84[0.25]$ \\
\cline { 1 - 4 } Weighted liver for analysis [mg] & $0.03[0.01]$ & $0.05[0.04]$ & $0.03[0]$ & $0.07[0.03]$ \\
\hline
\end{tabular}

P-values were calculated using exact permutations t-tests. *P-values $<0.05$ were considered significant (asterisk). CS- Cesarean section 
Table 2 Median [interquartile range] of selected metabolite concentrations in fetal plasma ( $\mu \mathrm{mol} / \mathrm{l})$ and fetal liver ( $\mu$ mol/kg) as well as metabolite sums and ratios of maternal nutrient restricted group (MNR) or control group (CTR) at 0.5 and 0.9 gestation (G)

\begin{tabular}{|c|c|c|c|c|c|c|c|c|c|}
\hline & \multirow{3}{*}{ Metabolite } & \multicolumn{3}{|c|}{ MNR } & \multicolumn{3}{|c|}{ CTR } & \multirow{2}{*}{$\frac{0.5 G}{p}$} & \multirow{2}{*}{$\frac{0.9 G}{p}$} \\
\hline & & $0.5 \mathrm{G}$ & $0.9 G$ & $\mathbf{p}$ & & $0.9 G$ & $\mathbf{p}$ & & \\
\hline & & $(\mathrm{n}=8)$ & $(n=4)$ & $\begin{array}{c}0.5 G \text { vs. } \\
\text { 0.9G }\end{array}$ & $(\mathrm{n}=6)$ & $(\mathrm{n}=9)$ & $\begin{array}{c}0.5 \text { vs. } \\
0.9 \mathrm{G}\end{array}$ & MNR vs. CTR & MNR vs. CTR \\
\hline \multirow{22}{*}{ 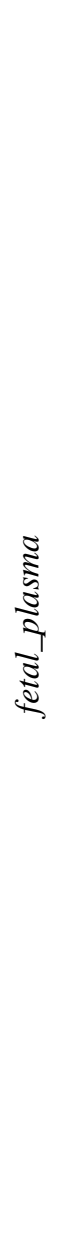 } & NEFA 26:5 & $0.004[0.001]$ & $0.002[0.001]$ & 0.127 & $0.004[0.001]$ & $0.003[0.001]$ & 0.897 & 0.642 & 0.036 \\
\hline & Met & $50.400[14.750]$ & $39.550[3.375]$ & 0.234 & $55.800[10.850]$ & $49.400[1.300]$ & 0.15 & 0.687 & 0.015 \\
\hline & Thr & $136.000[60.250]$ & 111.000 [11.750] & 0.075 & $146.000[26.250]$ & $167.000[40.000]$ & 0.679 & 0.874 & 0.022 \\
\hline & Carn C2 & 9.427 [2.697] & 5.248 [1.099] & 0.006 & $6.806[5.084]$ & $4.569[2.125]$ & 0.615 & 0.357 & 0.82 \\
\hline & Carn C3 & $1.100[0.488]$ & $0.531[0.158]$ & 0.008 & $0.666[0.271]$ & $0.310[0.165]$ & 0.119 & 0.014 & 0.761 \\
\hline & Carn C4 & $0.945[0.581]$ & $0.502[0.172]$ & 0.026 & $0.563[0.081]$ & $0.370[0.115]$ & 0.571 & 0.02 & 0.947 \\
\hline & Carn C5 & 0.370 [0.229] & $0.271[0.084]$ & 0.242 & $0.215[0.124]$ & $0.255[0.044]$ & 0.221 & 0.049 & 0.607 \\
\hline & Carn C6 & $0.050[0.019]$ & $0.029[0.004]$ & 0.028 & $0.026[0.020]$ & $0.029[0.028]$ & 0.626 & 0.015 & 0.667 \\
\hline & Carn C8 & $0.118[0.072]$ & 0.093 [0.019] & 0.127 & $0.062[0.052]$ & $0.136[0.092]$ & 0.224 & 0.029 & 0.538 \\
\hline & Carn C9 & $0.016[0.008]$ & $0.008[0.002]$ & 0.018 & $0.010[0.005]$ & $0.012[0.009]$ & 0.863 & 0.022 & 0.601 \\
\hline & lysoPC a C20:4 & $23.953[6.062]$ & $12.652[2.825]$ & 0.006 & $25.101[10.277]$ & $15.968[1.500]$ & 0.003 & 0.906 & 0.007 \\
\hline & PC aa C36:2 & 83.423 [11.855] & 88.283 [48.881] & 0.594 & $66.431[6.423]$ & 88.422 [21.145] & 0.038 & 0.032 & 0.951 \\
\hline & PC ae C36 & $0.521[0.206]$ & $0.466[0.232]$ & 1 & $0.360[0.085]$ & $0.500[0.089]$ & $<0.001$ & 0.048 & 0.745 \\
\hline & $\mathrm{PC}$ ae $\mathrm{C} 36: 2$ & $2.248[0.631]$ & 3.070 [1.167] & 0.093 & $1.797[0.137]$ & $3.036[0.904]$ & 0.001 & 0.034 & 0.945 \\
\hline & PC ae C38:2 & $0.414[0.044]$ & $0.613[0.046]$ & 0.002 & $0.244[0.051]$ & $0.580[0.134]$ & 0.001 & 0.006 & 0.354 \\
\hline & SM C34:2 & 9.966 [1.539] & 8.346 [2.918] & 0.188 & $7.310[1.820]$ & $8.660[2.850]$ & 0.234 & 0.043 & 0.583 \\
\hline & SM C36:2 & $6.082[0.864]$ & $7.748[2.836]$ & 0.057 & $4.850[0.643]$ & $7.664[0.920]$ & 0.002 & 0.024 & 0.827 \\
\hline & SM C42:3 & $14.220[2.720]$ & $18.105[4.400]$ & 0.18 & $11.119[2.535]$ & $17.349[0.617]$ & $<0.001$ & 0.018 & 1 \\
\hline & $\mathrm{SM}(\mathrm{OH}) \mathrm{C} 34: 2$ & $1.313[0.345]$ & 2.177 [1.029] & 0.02 & $1.053[0.183]$ & $1.929[0.650]$ & 0.001 & 0.015 & 0.73 \\
\hline & $\mathrm{SM}(\mathrm{OH}) \mathrm{C} 38: 2$ & $0.704[0.206]$ & $0.763[0.174]$ & 0.226 & $0.498[0.053]$ & $0.817[0.313]$ & 0.007 & 0.033 & 0.846 \\
\hline & Sum of AA & $\begin{array}{l}4253.069 \\
{[382.530]}\end{array}$ & $\begin{array}{l}3753.000 \\
{[486.237]}\end{array}$ & 0.143 & $\begin{array}{l}4571.827 \\
{[849.561]}\end{array}$ & $\begin{array}{l}3973.587 \\
{[198.575]}\end{array}$ & 0.032 & 0.727 & 0.113 \\
\hline & Sum of BCAA & $422.650[108.350]$ & $331.750[68.350]$ & 0.038 & $441.550[88.750]$ & $437.500[98.400]$ & 0.803 & 0.45 & 0.117 \\
\hline
\end{tabular}




\begin{tabular}{|c|c|c|c|c|c|c|c|c|c|}
\hline & Sum of Carn & $12.521[4.575]$ & $7.693[0.872]$ & 0.01 & $9.273[5.755]$ & $5.904[2.564]$ & 0.696 & 0.149 & 0.908 \\
\hline & Sum of short-chain Carn & $2.319[1.040]$ & $1.401[0.236]$ & 0.014 & $1.436[0.500]$ & $0.934[0.305]$ & 0.545 & 0.01 & 1 \\
\hline & Sum of PC aa & $\begin{array}{l}1207.025 \\
{[167.473]}\end{array}$ & $\begin{array}{c}813.218 \\
{[354.950]}\end{array}$ & 0.014 & $\begin{array}{c}985.453 \\
{[204.655]}\end{array}$ & 835.366 [164.265] & 0.017 & 0.246 & 0.635 \\
\hline & Sum of PC ae & 98.393 [8.757] & $94.628[39.558]$ & 0.741 & $79.356[11.852]$ & $105.833[13.434]$ & 0.005 & 0.109 & 0.427 \\
\hline & Sum of PC & $\begin{array}{c}1307.755 \\
{[173.290]}\end{array}$ & $\begin{array}{c}907.845 \\
{[394.508]}\end{array}$ & 0.028 & $\begin{array}{c}1061.247 \\
{[215.568]}\end{array}$ & 946.796 [173.073] & 0.041 & 0.226 & 0.635 \\
\hline & Sum of SM & $174.428[32.313]$ & $182.382[77.451]$ & 0.535 & $139.209[21.214]$ & 175.604 [20.397] & 0.003 & 0.087 & 0.834 \\
\hline & Ratio Carn C4/Val & $0.003[0.001]$ & $0.002[0.001]$ & 0.145 & $0.002[0.001]$ & $0.002[0.001]$ & 0.37 & 0.019 & 0.303 \\
\hline & Ratio Carn/BCAA & $0.005[0.002]$ & $0.004[0.001]$ & 0.135 & $0.004[0.001]$ & $0.002[0.002]$ & 0.303 & 0.037 & 0.288 \\
\hline & Ratio Carn C3/Met & $0.022[0.016]$ & $0.013[0.003]$ & 0.101 & $0.014[0.005]$ & $0.006[0.005]$ & 0.227 & 0.046 & 0.348 \\
\hline & Ratio Carn C3/Thr & $0.007[0.004]$ & $0.005[0.001]$ & 0.081 & $0.004[0.001]$ & $0.002[0.001]$ & 0.121 & 0.042 & 0.182 \\
\hline & Ratio Carn C3/Ile & $0.012[0.007]$ & $0.008[0.002]$ & 0.061 & $0.008[0.003]$ & $0.004[0.004]$ & 0.062 & 0.048 & 0.175 \\
\hline & & $\mathrm{n}=8$ & $\mathrm{n}=3$ & & $\mathrm{n}=5$ & $\mathrm{n}=9$ & & & \\
\hline & NEFA 26:1 & $0.013[0.004]$ & $0.001[0.000]$ & 0.017 & $0.013[0.002]$ & $0.004[0.002]$ & $<0.001$ & 0.679 & 0.036 \\
\hline & Thr & 299.259 [63.197] & $175.127[15.043]$ & 0.012 & $\begin{array}{c}342.746 \\
{[164.617]}\end{array}$ & $278.571[71.754]$ & 1 & 0.845 & 0.009 \\
\hline & Carn C10:1 & $0.002[0.001]$ & $0.002[0.001]$ & 0.317 & $0.001[0.001]$ & $0.001[0.002]$ & 0.263 & $\mathbf{0 . 0 3}$ & 0.786 \\
\hline & Carn C12:1 & $0.004[0.002]$ & $0.031[0.010]$ & 0.008 & $0.004[0.002]$ & $0.009[0.014]$ & 0.057 & 0.673 & 0.027 \\
\hline & $\mathrm{PC}$ aa $\mathrm{C} 30$ & $1.630[0.232]$ & $0.493[0.216]$ & 0.008 & $1.114[0.262]$ & $0.599[0.216]$ & 0.003 & 0.036 & 0.841 \\
\hline & PC aa C34:1 & $17.322[3.595]$ & 16.812 [1.950] & 0.683 & $16.379[1.261]$ & $12.394[4.874]$ & 0.02 & 0.93 & 0.023 \\
\hline 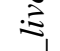 & $\mathrm{PC}$ aa $\mathrm{C} 38: 3$ & $3.317[0.882]$ & $6.257[0.785]$ & 0.008 & $2.972[0.349]$ & $4.344[1.720]$ & 0.124 & 0.57 & 0.041 \\
\hline $\mathbb{\Xi}$ & $\mathrm{PC}$ ae $\mathrm{C} 34$ & $0.185[0.017]$ & $0.111[0.057]$ & 0.042 & $0.144[0.045]$ & $0.088[0.026]$ & 0.018 & 0.006 & 0.364 \\
\hline & $\mathrm{PC}$ ae $\mathrm{C} 34: 1$ & $1.043[0.248]$ & $0.970[0.145]$ & 0.842 & $0.937[0.087]$ & $0.651[0.165]$ & 0.001 & 0.791 & 0.009 \\
\hline & PC ae C44:4 & $0.002[0.001]$ & $0.007[0.002]$ & $\mathbf{0 . 0 3 3}$ & $0.005[0.001]$ & $0.004[0.001]$ & 0.365 & 0.2 & $\mathbf{0 . 0 2 3}$ \\
\hline & $\mathrm{SM}(\mathrm{OH}) \mathrm{C} 40: 2$ & $0.161[0.035]$ & $0.163[0.067]$ & 0.492 & $0.112[0.025]$ & $0.162[0.077]$ & 0.351 & 0.042 & 0.805 \\
\hline & $\mathrm{SM}(\mathrm{OH}) \mathrm{C} 42: 4$ & $0.009[0.008]$ & $0.025[0.003]$ & 0.008 & $0.010[0.001]$ & $0.012[0.007]$ & 0.554 & 0.624 & 0.005 \\
\hline & Sum of AA & $\begin{array}{c}9112.057 \\
{[2226.284]}\end{array}$ & $\begin{array}{c}6326.997 \\
{[597.671]}\end{array}$ & 0.024 & $\begin{array}{c}8965.837 \\
{[302.529]}\end{array}$ & $\begin{array}{c}7024.026 \\
{[1156.955]}\end{array}$ & 0.022 & 0.88 & 0.195 \\
\hline & Sum of BCAA & $343.306[50.721]$ & 340.102 [32.715] & 1 & $290.435[57.233]$ & 410.565 [86.167] & 0.05 & 0.671 & 0.141 \\
\hline & Sum of short-chain Carn & $0.593[0.315]$ & $1.598[0.076]$ & 0.006 & $0.306[0.238]$ & $0.956[1.188]$ & 0.096 & 0.786 & 0.3 \\
\hline
\end{tabular}




\begin{tabular}{|c|c|c|c|c|c|c|c|c|}
\hline Sum of lyso PC a & $2.459[0.344]$ & 6.571 [1.589] & 0.006 & $2.202[0.404]$ & 4.787 [2.951] & 0.015 & 0.823 & 0.532 \\
\hline Sum of PC aa & $128.116[22.541]$ & $252.448[36.982]$ & 0.006 & $123.978[19.074]$ & 193.528 [109.324] & 0.081 & 0.879 & 0.2 \\
\hline Sum of PC ae & 9.923 [2.120] & $18.161[4.314]$ & 0.012 & 8.774 [2.406] & $13.133[8.921]$ & 0.126 & 0.78 & 0.218 \\
\hline Sum of PC & $138.039[24.660]$ & 273.597 [39.802] & 0.006 & $132.606[21.334]$ & $206.661[118.241]$ & 0.099 & 0.879 & 0.2 \\
\hline Sum of SM & 15.525 [4.092] & $19.650[5.593]$ & 0.03 & 13.696 [1.891] & $17.741[6.425]$ & 0.074 & 0.542 & 0.109 \\
\hline Ratio Carn C5/Leu & $0.000[0.000]$ & $0.000[0.000]$ & 0.308 & $0.000[0.000]$ & $0.000[0.000]$ & 0.856 & 0.045 & 0.114 \\
\hline Ratio Carn/BCAA & $0.002[0.001]$ & $0.005[0.001]$ & 0.006 & $0.001[0.001]$ & $0.003[0.003]$ & 0.211 & 0.958 & 0.059 \\
\hline Ratio Cys/Met & 2.997 [2.154] & $5.990[3.079]$ & 1 & $1.946[1.611]$ & $2.070[1.080]$ & 0.793 & 0.848 & 0.05 \\
\hline Ratio Carn C3/Met & $0.024[0.011]$ & $0.090[0.018]$ & 0.008 & $0.014[0.012]$ & $0.050[0.053]$ & 0.186 & 0.952 & 0.041 \\
\hline Ratio Carn C3/Thr & $0.002[0.001]$ & $0.009[0.001]$ & 0.008 & $0.002[0.001]$ & $0.002[0.004]$ & 0.337 & 0.73 & 0.023 \\
\hline Ratio Carn C3/Ile & $0.006[0.002]$ & $0.019[0.002]$ & 0.008 & $0.004[0.005]$ & $0.011[0.017]$ & 0.217 & 0.855 & 0.173 \\
\hline Ratio Carn C3/Val & $0.004[0.001]$ & $0.012[0.001]$ & 0.008 & $0.004[0.004]$ & 0.007 [0.007] & 0.336 & 0.515 & 0.041 \\
\hline Ratio Carn C3/Ser & $0.000[0.000]$ & $0.003[0.001]$ & 0.008 & $0.000[0.000]$ & $0.001[0.002]$ & 0.161 & 0.797 & 0.155 \\
\hline
\end{tabular}

3 P-values were calculated using permutation tests. P-values < 0.05 were considered significant (bold). Abbreviations: AA, amino acids; BCAA, branched-chain amino acids; NEFA, non-esterified fatty acids; lysoPC a, lysophosphatidylcholines; PC aa phosphatidylcholines; PC ae, ether-linked phosphatidylcholines; SM, sphingomyelis 